\title{
HODGE THEORY AND LAGRANGIAN PLANES ON GENERALIZED KUMMER FOURFOLDS
}

\author{
BRENDAN HASSETT AND YURI TSCHINKEL
}

\section{INTRODUCTION}

Suppose $X$ is a smooth projective complex variety. Let $\mathrm{N}_{1}(X, \mathbb{Z}) \subset$ $\mathrm{H}_{2}(X, \mathbb{Z})$ and $\mathrm{N}^{1}(X, \mathbb{Z}) \subset \mathrm{H}^{2}(X, \mathbb{Z})$ denote the group of curve classes modulo homological equivalence and the Néron-Severi group respectively. The monoids of effective classes in each group generate cones $\mathrm{NE}_{1}(X) \subset \mathrm{N}_{1}(X, \mathbb{R})$ and $\mathrm{NE}^{1}(X) \subset \mathrm{N}^{1}(X, \mathbb{R})$ with closures $\overline{\mathrm{NE}}_{1}(X)$ and $\overline{\mathrm{NE}}^{1}(X)$, the pseudoeffective cones. These play a central rôle in the birational geometry of $X$.

Let $X$ be an irreducible holomorphic symplectic variety, i.e., a smooth projective simply-connected manifold admitting a unique nondegenerate holomorphic two-form. Let (,) denote the Beauville-Bogomolov form on the cohomology group $\mathrm{H}^{2}(X, \mathbb{Z})$, normalized so that it is integral and primitive. Duality gives a $\mathbb{Q}$-valued form on $\mathrm{H}_{2}(X, \mathbb{Z})$, also denoted (, ). When $X$ is a $\mathrm{K} 3$ surface these coincide with the intersection form. In higher-dimensions, the form induces an inclusion

$$
\mathrm{H}^{2}(X, \mathbb{Z}) \subset \mathrm{H}_{2}(X, \mathbb{Z}),
$$

which allows us to extend $($,$) to a \mathbb{Q}$-valued quadratic form.

Now suppose that $X$ contains a Lagrangian projective space $\mathbb{P}^{\operatorname{dim}(X) / 2}$; let $\ell \in \mathrm{H}_{2}(X, \mathbb{Z})$ denote the class of a line in $\mathbb{P}^{\operatorname{dim}(X) / 2}$, and $\lambda=N \ell \in$ $\mathrm{H}^{2}(X, \mathbb{Z})$ a positive integer multiple; we can take $N$ to be the index of $\mathrm{H}^{2}(X, \mathbb{Z}) \subset \mathrm{H}_{2}(X, \mathbb{Z})$. Hodge theory 27,33 shows that the deformations of $X$ containing a deformation of the Lagrangian space coincide with the deformations of $X$ for which $\lambda \in \mathrm{H}^{2}(X, \mathbb{Z})$ remains of type $(1,1)$. Infinitesimal Torelli implies this is a divisor on the deformation space, i.e.,

$$
\lambda^{\perp} \subset \mathrm{H}^{1}\left(X, \Omega_{X}^{1}\right) \simeq \mathrm{H}^{1}\left(X, T_{X}\right) .
$$

Our goal is to establish intersection theoretic properties of $\ell$ for various deformation-equivalence classes of holomorphic symplectic varieties. Previous results in this direction include

(1) If $X$ is a $\mathrm{K} 3$ surface then $(\ell, \ell)=-2$.

Date: October 31, 2018. 
(2) If $X$ is deformation equivalent to the Hilbert scheme of length two subschemes of a K3 surface then $(\ell, \ell)=-5 / 2$ [15].

Here we prove

Theorem 1.1. If $X$ is deformation equivalent to the generalized Kummer manifold of dimension four and $\ell$ the class of a line on a Lagrangian plane in $X$ then

$$
(\ell, \ell)=-3 / 2 \text {. }
$$

In Section 14, we discuss divisibility properties of $\ell$ and the orbit of $[\ell]$ under the monodromy action.

This is part of a program described in [14] to characterize numerically classes of extremal rays in holomorphic symplectic manifolds, with a view towards characterizing ample divisors in terms of the intersection properties of the Beauville-Bogomolov form on the divisor/curve classes. We expect that geometric properties of birational contractions should be encoded in the self-intersections of their extremal rational curves. In the examples we have considered, extremal rays associated to Lagrangian projective spaces have smallest self-intersection. See [13, 15] for a detailed discussion of Hilbert schemes of length-two subschemes of K3 surfaces. Markman [24] addresses divisorial contractions and the associated reflections.

The main ingredients in our proof include an analysis of automorphisms of generalized Kummer varieties, their fixed-point loci, and the resulting 'tautological' Hodge classes in middle cohomology. (Unlike the case of length-two Hilbert schemes, the middle cohomology is not generated by the second cohomology.) In particular, these tautological classes arise from explicit complex surfaces (see Theorem 4.4). We analyze the saturation of the lattice generated by these tautological classes in the middle cohomology, and integrality properties of the quadratic form associated with the cup product. Computing the orbit of a Lagrangian plane under the automorphism group, we obtain a precise characterization of the homology classes that may arise (see Proposition 11.1 and Theorem 11.2).

Acknowledgments: We are grateful to Lothar Göttsche, Daniel Huybrechts, János Kollár, Marc Nieper-Wisskirchen, and Justin Sawon for useful conversations and correspondence. The first author was supported by National Science Foundation Grant 0554491 and 0901645; the second author was supported by National Science Foundation Grants 0554280 and 0602333. We appreciate the hospitality of the American Institute of Mathematics, where some of this work was done. 


\section{AutomorPhisms of HOLOMORPHIC SYMPLECTIC MANIFOLDS}

Let $X$ be an irreducible holomorphic symplectic manifold. Let

$$
\operatorname{Aut}^{\circ}(X) \subset \operatorname{Aut}(X)
$$

denote the subgroup of holomorphic automorphisms of $X$ acting trivially on $\mathrm{H}^{1}\left(T_{X}\right)$ and preserving the symplectic form $\omega$. Since $X$ has no vector fields, this is a discrete group. This is equivalent to the automorphisms acting trivially on $\mathrm{H}^{2}(X, \mathbb{C})$, as

$$
\begin{aligned}
\mathrm{H}^{2}(X, \mathbb{C}) & =\mathrm{H}^{2}\left(\mathcal{O}_{X}\right) \oplus \mathrm{H}^{1}\left(\Omega_{X}^{1}\right) \oplus \mathrm{H}^{0}\left(\Omega_{X}^{2}\right) \\
& =\overline{\mathbb{C} \omega} \oplus\left(\omega \otimes \mathrm{H}^{1}\left(T_{X}\right)\right) \oplus \mathbb{C} \omega .
\end{aligned}
$$

Let $X^{\prime}$ be deformation equivalent to $X$, i.e., there exists a connected complex manifold $B$, with distinguished points $b$ and $b^{\prime}$, and a proper family of complex manifolds $\pi: \mathcal{X} \rightarrow B$ with $\mathcal{X}_{b}:=\pi^{-1}(b)=X$ and $\mathcal{X}_{b^{\prime}}=\pi^{-1}\left(b^{\prime}\right)=X^{\prime}$.

Theorem 2.1. $\operatorname{Aut}^{\circ}(X)$ is a deformation invariant of $X$, i.e., there exists a local system of groups

$$
\operatorname{Aut}^{\circ}(\mathcal{X} / B) \rightarrow B
$$

acting on $\mathcal{X} \rightarrow B$, such that for each $b^{\prime} \in B$ the fiber is isomorphic to $\operatorname{Aut}^{\circ}\left(X^{\prime}\right)$.

Proof. Consider a local universal deformation space of $X$

$$
\psi: \mathcal{U} \rightarrow \Delta
$$

where $\Delta$ is a small polydisk and $\mathcal{U}_{0}=X[19$. The completeness of this family implies we can construct this equivariantly for the action of $\operatorname{Aut}(X)$, i.e., $\operatorname{Aut}(X)$ acts on $\mathcal{U}$ and $\Delta$ and $\psi$ is equivariant with respect to these actions. However, $\operatorname{Aut}^{\circ}(X)$ acts trivially on the tangent space $T_{0} \Delta=\mathrm{H}^{1}\left(T_{X}\right)$, thus acts trivially on $\Delta$ as well. It follows that $\operatorname{Aut}^{\circ}(X)$ acts fiberwise on $\mathcal{U} \rightarrow \Delta$.

Let $\operatorname{Aut}^{\circ}(\mathcal{X} / B)$ denote the group over $B$ classifying automorphisms acting trivially on $\mathrm{H}^{2}\left(\mathcal{X}_{b}\right)$ for each $b \in B$. The previous analysis shows $\operatorname{Aut}^{\circ}(\mathcal{X} / B) \rightarrow B$ is a local homeomorphism. It remains to show this is universally closed.

We first show that each automorphism specializes to a bimeromorphic mapping $\phi: X^{\prime} \rightarrow X^{\prime}$. The reasoning is identical to the proof of [16, 4.3], i.e., that two non-separated points in the moduli space correspond to bimeromorphic holomorphic symplectic manifolds. Consider a convergent sequence $b_{n} \rightarrow b^{\prime}$ and a sequence $\alpha_{n} \in \operatorname{Aut}^{\circ}\left(\mathcal{X}_{b_{n}}\right)$. The sequence of graphs

$$
\Gamma_{n}:=\Gamma_{\alpha_{n}} \subset \mathcal{X}_{b_{n}} \times \mathcal{X}_{b_{n}}
$$


admits a convergent subsequence. Let $\Gamma^{\prime} \subset X^{\prime} \times X^{\prime}$ denote the resulting limit cycle, which necessarily includes a component $Z$ that maps bimeromorphically to each factor. We take $\phi$ to be the bimeromorphic map associated with $Z$.

The second step is to show that

$$
\phi_{*}: \mathrm{H}^{2}\left(X^{\prime}, \mathbb{Z}\right) \rightarrow \mathrm{H}^{2}\left(X^{\prime}, \mathbb{Z}\right)
$$

is the identity. This essentially follows from the description of the 'birational Kähler cone' in [17, §4], but we offer a proof here. Since $\Gamma^{\prime}$ is the specialization of a correspondence acting as the identity on $\mathrm{H}^{2}$, we know that $\Gamma_{*}^{\prime}=$ Id. Express

$$
\Gamma^{\prime}=Z+\sum_{i} Y_{i} \subset X^{\prime} \times X^{\prime}
$$

where the $Y_{i}$ map to proper analytic subsets of each factor; let $\pi_{1}$ and $\pi_{2}$ denote the projections. It suffices to show that $\pi_{j}\left(Y_{i}\right), j=1,2$, has codimension $>1$; then $Y_{i}$ acts trivially on $\mathrm{H}^{2}$ and $\phi_{*}=\Gamma_{*}^{\prime}=\mathrm{Id}$ on $\mathrm{H}^{2}$. Since $\phi$ is a bimeromorphic map from a holomorphic symplectic manifold to itself, it is an isomorphism in codimension one and $\phi_{*}$ is an isomorphism of $\mathrm{H}^{2}$. Enumerate the $Y_{i}$ such that $\pi_{1}\left(Y_{i}\right)$ has codimension one, i.e., $Y_{1}, \ldots, Y_{k}$; since $\phi$ is an isomorphism in codimension one, these coincide with the components such that $\pi_{2}\left(Y_{i}\right)$ has codimension one (cf. the proofs of [17, 2.5,4.2]). Furthermore, $\phi$ respects these divisors in the sense

$$
\phi_{*}\left(\sum_{i=1}^{k} \pi_{1}\left(Y_{i}\right)\right)=\sum_{i=1}^{k} \pi_{2}\left(Y_{i}\right)
$$

indeed, $Y_{i}$ is ruled over both $\pi_{1}\left(Y_{i}\right)$ and $\pi_{2}\left(Y_{i}\right)$. In this situation, we have [17, p. 508]

$$
\sum_{i=1}^{k}\left[Y_{i}\right]_{*}\left(\sum_{i=1}^{k} \pi_{1}\left(Y_{i}\right)\right)=-\sum_{i=1}^{k} b_{i} \pi_{2}\left(Y_{i}\right), \quad b_{i}>0 .
$$

The previous two equations contradict the fact that $\Gamma^{\prime}$ introduces the identity on the second homology.

Thus $\phi$ is a bimeromorphic mapping of $K$-trivial varieties respecting Kähler cones. A result of Fujiki [9] implies $\phi$ is an isomorphism.

Remark 2.2. This is related to unpublished results of Kaledin and Verbitsky [18, §6], where such automorphisms of generalized Kummer varieties $K_{n}(A)$ were used to exhibit nontrivial trianalytic subvarieties of $K_{n}(A)$. 


\section{Application to Generalized Kummer varieties}

Suppose that $X$ is a holomorphic symplectic variety of dimension $2 n$, deformation equivalent to a generalized Kummer variety $K_{n}(A)$, defined as follows: Given an abelian surface $A$ with degree $(n+1)$ Hilbert scheme $A^{[n+1]}, K_{n}(A)$ is the fiber over 0 of the addition map $\alpha: A^{[n+1]} \rightarrow A$. The Beauville-Bogomolov form is given by

$$
\mathrm{H}^{2}\left(K_{n}(A), \mathbb{Z}\right)=\mathrm{H}^{2}(A, \mathbb{Z}) \oplus_{\perp} \mathbb{Z} e, \quad(e, e)=-2(n+1),
$$

where $2 e$ is the class $E$ of the nonreduced subschemes [34, §4.3.1]. Each class $\eta \in \mathrm{H}^{2}(A, \mathbb{Z})$ yields a class in $\mathrm{H}^{2}\left(K_{n}(A), \mathbb{Z}\right)$, i.e., the subschemes with some support along $\eta$. We use $($,$) to embed \mathrm{H}^{2}(X, \mathbb{Z}) \subset \mathrm{H}_{2}(X, \mathbb{Z})$ and extend $($,$) to a \mathbb{Q}$-valued form on $\mathrm{H}_{2}(X, \mathbb{Z})$. Let $e^{\vee} \in \mathrm{H}_{2}\left(K_{n}(A), \mathbb{Z}\right)$ denote the class of a general ruling of $E$, i.e., where $n-1$ of the points are fixed and the tangent vector at the $n$th point varies. We have

$$
e=2(n+1) e^{\vee}, \quad\left(e^{\vee}, e^{\vee}\right)=-\frac{1}{2(n+1)} .
$$

The following data can be extracted from Göttsche's generating series for the cohomology of Kummer varieties [11, p. 50]. The table displays the Betti numbers $\beta_{\nu}\left(K_{n}(A)\right)$ for $n \leq 4$ :

\begin{tabular}{c|cccc}
$\quad n$ & 1 & 2 & 3 & 4 \\
$\nu$ & & & & \\
\hline 0 & 1 & 1 & 1 & 1 \\
1 & 0 & 0 & 0 & 0 \\
2 & 22 & 7 & 7 & 7 \\
3 & 0 & 8 & 8 & 8 \\
4 & 1 & 108 & 51 & 36 \\
5 & & 8 & 56 & 64 \\
6 & & 7 & 458 & 168 \\
7 & & 0 & 56 & 288 \\
8 & & 1 & 51 & 1046
\end{tabular}

Consider the group

$$
G_{n}=\mathbb{Z} / 2 \mathbb{Z} \ltimes(\mathbb{Z} / n \mathbb{Z})^{4}
$$

where $\mathbb{Z} / 2 \mathbb{Z}$ acts on $(\mathbb{Z} / n \mathbb{Z})^{4}$ via \pm 1 . Let $A$ be an abelian surface and identify $A[n]=(\mathbb{Z} / n \mathbb{Z})^{4}$. Then $A[n]$ acts on $A$ via translation and $\mathbb{Z} / 2 \mathbb{Z}$ acts on $A$ via \pm 1 . Altogether, we get an action

$$
G_{n} \times A \rightarrow A
$$

Note that the induced action is trivial on $\mathrm{H}^{2}(A)$. Conversely, any finite group acting on $A$ such that the induced action on $\mathrm{H}^{2}(A)$ is trivial is a subgroup of $G_{n}$ for some $n$. Indeed, actions fixing 0 are determined by 
the associated representation on $\mathrm{H}^{1}(A)$, but the only group acting on $\mathrm{H}^{1}(A)$ such that the induced action on $\bigwedge^{2}\left(\mathrm{H}^{1}(A)\right)=\mathrm{H}^{2}(A)$ is trivial is \pm 1 .

Theorem 2.1 and the description on the second cohomology of generalized Kummer manifolds yields:

Proposition 3.1. For each $n>2$, the action of $G_{n}$ on $A$ induces a natural action on $K_{n-1}(A)$, which is trivial on $\mathrm{H}^{2}\left(K_{n-1}(A)\right)$. This action extends to a natural action on any deformation $X$ on $K_{n-1}(A)$.

The action of $G_{n}$ on $K_{n-1}(A)$ has been considered previously, e.g., [28, 3].

\section{Analysis of the Cohomology of $K_{2}(A)$}

We recall the structure of the cohomology ring of $K_{2}(A)$, following [31], [20, §4], [26], and [28]:

Proposition 4.1. [20, 4.6] Let $X$ be deformation equivalent to $K_{2}(A)$ for $A$ an abelian surface. The Lie algebra $\mathfrak{s o}(4,5)$ acts on the cohomology $\mathrm{H}^{*}(X)$ which admits a decomposition

$$
\mathrm{H}^{*}(X)=\operatorname{Sym}\left(\mathrm{H}^{2}(X)\right) \oplus \mathbf{1}_{X}^{80} \oplus\left(\mathrm{H}^{3}(X) \oplus \mathrm{H}^{5}(X)\right),
$$

where the middle term is a trivial $\mathfrak{s o}(4,5)$-representation consisting of Hodge cycles in $\mathrm{H}^{4}(X)$ and the last term carries the structure of a sixteen-dimensional spinor representation.

Furthermore, the induced decomposition on middle cohomology

$$
\mathrm{H}^{4}(X)=\operatorname{Sym}^{2}\left(\mathrm{H}^{2}(X)\right) \oplus_{\perp} \mathbf{1}_{X}^{80}
$$

is orthogonal under the intersection form.

Decomposition (2) is not the only natural decomposition of the middle cohomology. The second Chern class $c_{2}(X)$ is also an invariant element of $\mathrm{H}^{4}(X)$ but is not orthogonal to $\operatorname{Sym}^{2}\left(\mathrm{H}^{2}(X)\right)$ [20, 4.8]; indeed, we have the Fujiki relation

$$
c_{2}(X) \cdot D_{1} \cdot D_{2}=a_{X}\left(D_{1}, D_{2}\right), \quad D_{1}, D_{2} \in H^{2}(X),
$$

where $a_{X}$ is a nonzero constant (which will be computed explicitly below). Writing

$$
\operatorname{Sym}^{2}\left(\mathrm{H}^{2}(X)\right)^{\circ}=c_{2}(X)^{\perp} \cap \operatorname{Sym}^{2}\left(\mathrm{H}^{2}(X)\right),
$$

we obtain an alternate decomposition

$$
\mathrm{H}^{4}(X)=\operatorname{Sym}^{2}\left(\mathrm{H}^{2}(X)\right)^{\circ} \oplus_{\perp} \mathbf{1}_{X}^{81} .
$$

By the infinitesimal Torelli theorem, the first summand contains no Hodge classes for general deformations $X$. Indeed, since the period map 
is a local diffeomorphism, $\mathrm{H}^{2}(X)$ carries a general weight-two Hodge structure with the relevant numerical invariants; such a Hodge structure is Mumford-Tate generic [32, §2]. Hodge cycles in $\operatorname{Sym}^{2}\left(\mathrm{H}^{2}(X)\right)$ yield invariants for the action of the Hodge group of $\mathrm{H}^{2}(X)$, which is the special orthogonal group for $\mathrm{H}^{2}(X)$ under the Beauville-Bogomolov form. The only such invariants in $\operatorname{Sym}^{2}\left(\mathrm{H}^{2}(X)\right)$ are multiples of the dual to the Beauville-Bogomolov form.

Integral classes in the summand $\mathbf{1}_{X}^{81}$ are called canonical Hodge classes, because they remain of type $(2,2)$ under arbitrary deformations.

We construct 81 distinguished rational surfaces in $X=K_{2}(A)$, whose classes span an 81-dimensional subspace in $\mathrm{H}^{4}(X, \mathbb{Q})$ which contains the summand $\mathbf{1}_{X}^{80}$ above. This 81-dimensional space is different from the subspace indicated in (3), but we will describe explicitly how they are related.

For each $\tau \in A$, let $W_{\tau}$ denote the subschemes in $A^{[3]}$ supported entirely at $\tau$, with the induced reduced scheme structure. Briançon [4, p. 76] gives explicit equations for the corresponding subscheme of the Hilbert scheme, via local coordinates. Eliminating embedded components from these equations, we find that $W_{\tau} \simeq \mathbb{P}(1,1,3)$, realized as a cone over a twisted cubic in $\mathbb{P}^{4}$.

In the case where $\tau \in A[3]$, we have $W_{\tau} \subset K_{2}(A)$. This yields 81 disjoint copies of $\mathbb{P}(1,1,3)$. We recover some of their intersection properties:

- $W_{\tau}^{2}=3$;

- $W_{\tau} e^{2}=3$

- $W_{\tau} c_{2}\left(K_{2}(A)\right)=-1$.

We prove these assertions. Using the fibration

$$
\begin{aligned}
K_{2}(A) \rightarrow & A^{[3]} \\
& \downarrow \Sigma \\
& A
\end{aligned}
$$

we can reduce the computation of the first number to the main result of [8]. For the second number, it suffices to check that

$$
\mathcal{O}_{K_{2}(A)}(e) \mid W_{\tau}=\mathcal{O}_{\mathbb{P}(1,1,3)}(-H)
$$

where $H$ is the hyperplane class associated with $\mathbb{P}(1,1,3) \hookrightarrow \mathbb{P}^{4}$. Let $R$ denote the extremal ray corresponding to the generic fiber of the diagonal divisor (in $K_{2}(A)$ ) over the diagonal of the symmetric product. The diagonal divisor has class $2 e$ and $2 e \cdot R=-2$, as the symmetric product has $A_{1}$-singularities at the generic point of the diagonal. Thus $e \cdot R=-1$. However, $R$ specializes to some cycle of rational curves 
$R_{0} \subset W_{\tau}$ and $e \mid W_{\tau}$ is a nontrivial Cartier divisor, i.e., $e=-n H$ for some $n$. Thus

$$
-1=e \cdot R=-n H \cdot R_{0},
$$

hence $n=1$ and $R_{0}$ is a ruling of $\mathbb{P}(1,1,3)$. For the third number, consider the induced morphism

$$
\phi: \mathbb{F}_{3} \rightarrow \mathbb{P}(1,1,3) \simeq W_{\tau} \hookrightarrow K_{2}(A)
$$

from the Hirzebruch surface resolving $W_{\tau}$; let $\Sigma_{0}$ denote the $(-3)$-curve contracted by $\phi$. We have the exact sequence

$$
0 \rightarrow T_{\mathbb{F}_{3}} \rightarrow \phi^{*} T_{K_{2}(A)} \rightarrow N_{\phi} \rightarrow 0
$$

which implies

$$
c_{2}\left(\phi^{*} T_{K_{2}(A)}\right)=c_{2}\left(N_{\phi}\right)-4
$$

We may interpret

$c_{2}\left(N_{\phi}\right)+\left\{\right.$ contribution of $\Sigma_{0}$ to the double point formula $\}=W_{\tau}^{2}=3$,

and an excess-intersection computation [10, ch. 9] shows that contribution of $\Sigma_{0}$ is zero. We conclude that $c_{2}\left(\phi^{*} T_{K_{2}(A)}\right)=-1$.

Remark 4.2 (contributed by M. Nieper-Wisskirchen). These computations can also be put into the framework of Nakajima's [25] description of the cohomology of the Hilbert scheme.

Consider the Galois cover

$$
\begin{aligned}
v: A \times K^{n-1}(A) & \rightarrow A^{[n]} \\
(a, \xi) & \mapsto a+\xi
\end{aligned}
$$

where $a+\xi$ denotes the translate of $\xi$ by $a$. The Galois group is $A[n]$. Let $B_{0}$ be the Briançon variety in $A^{[n]}$ of those subschemes whose support is 0 . Then $v^{*} B_{0}$ is the same as $0 \times W$ in the cohomology ring of $A \times K^{n-1}(A)$, where $W=\sum_{\tau \in A[n]} W_{\tau}$. Let $B$ be the Briançon variety in $A^{[n]}$ of those subschemes that are supported at a single arbitrary point. Then $v^{*} B=A \times W$.

Now assume $n=3$. To show that $W_{\tau}^{2}=3$, we have to show $\left(v^{*} B_{0}\right)$. $\left(v^{*} B\right)=81 \cdot 3$, as the different $W_{\tau}$ are orthogonal. As $\operatorname{deg}(v)=81$, this is the same as showing that $B_{0} \cdot B=3$ on the Hilbert scheme. Now $B_{0}$ happens to be $q_{3}(\omega)|\mathbf{0}\rangle$, where $\omega$ is the codimension-four class of a point in $A$, and $B$ is $q_{3}(1)|\mathbf{0}\rangle$, using the standard notation for the cohomology classes in the Nakajima basis [2, §4]. Using the wellknown commutation relations between these operators, one gets that the Poincaré duality pairing of $q_{3}(\omega)|\mathbf{0}\rangle$ and $q_{3}(1)|\mathbf{0}\rangle$ on the Hilbert scheme is exactly 3 .

Intersections with $c_{2}$ can be obtained using the techniques of [1, 2]. 
Consider the averaged class

$$
W:=\sum_{\tau \in A[3]} W_{\tau}
$$

with intersection properties

- $W^{2}=81 \cdot 3=243$;

- $W e^{2}=243$;

- $W c_{2}\left(K_{2}(A)\right)=-81$.

It follows that

$$
W=\frac{3}{8}\left(c_{2}(X)+3 e^{2}\right) .
$$

Given $p \in A$ consider the locus

$$
Y_{p}=\left\{\left(a_{1}, a_{2}, p\right): a_{1}+a_{2}+p=0\right\} \subset K_{2}(A) .
$$

Exchanging the first two terms induces

$$
\iota: a_{1} \mapsto-p-a_{1}
$$

on the first factor, which is conjugate to the sign involution. (Set $a_{1}=q+\alpha$ for $2 q=-p$; then $\iota(\alpha)=-\alpha$.) In addition, $Y_{p}$ meets the boundary at $\{(-2 p)+p+p\}$. Thus $Y_{p}$ is isomorphic to the Kummer surface $K_{1}(A)$ blown up at one point, i.e., the images of $\alpha= \pm 3 q$.

The diagonal divisor meets $Y_{p}$ along the 16 distinguished $(-2)$-curves and with multiplicity two along the $(-1)$-curve over the center, thus we have

$$
e^{2} Y_{p}=\frac{1}{4}(-4+16 \cdot(-2))=-9 .
$$

Evidently, $Y_{p}$ is disjoint from $W_{\tau}$ for $p \notin A[3]$, thus

$$
Y_{p} \cdot W_{\tau}=0 .
$$

Using the computations for $W_{\tau}$ done previously, we find

$$
Y_{p}=\frac{1}{72}\left(3 c_{2}\left(K_{2}(A)\right)+e^{2}\right) \text {. }
$$

In particular, $Y_{p}^{2}=1$ and given $f, g \in \mathrm{H}^{2}(A, \mathbb{C})$ we have

$$
f \cdot g \cdot Y_{p}=2 f \cdot g .
$$

Assume $p \notin A[3]$ and write

$$
Z_{\tau}=Y_{p}-W_{\tau} .
$$

As a consequence, we deduce

$$
Z_{\tau} \cdot D_{1} \cdot D_{2}=2\left(D_{1}, D_{2}\right)
$$


for all $D_{1}, D_{2} \in \mathrm{H}^{2}\left(K_{2}(A), \mathbb{Z}\right)$. By the orthogonal decompositions (2) and (3), we conclude $Z_{\tau} \in \mathbf{1}_{K_{2}(A)}^{81}$, i.e., they are canonical Hodge cycles. We summarize this analysis as follows:

Proposition 4.3. Let $X$ be deformation equivalent to $K_{2}(A)$, for $A$ an abelian surface. Consider the lattice of canonical Hodge classes

$$
\left(\mathbf{1}_{X}^{81} \cap \mathrm{H}^{4}(X, \mathbb{Z})\right) \subset \mathrm{H}^{4}(X, \mathbb{Z}),
$$

associated with the decomposition (3). The classes $\left\{Z_{\tau}\right\}_{\tau \in A[3]}$ in this lattice span $\left(\mathbf{1}_{X}^{81} \cap \mathrm{H}^{4}(X, \mathbb{Q})\right)$ and satisfy

$$
Z_{\tau}^{2}=4, \quad Z_{\tau} \cdot Z_{\tau^{\prime}}=1 \text { if } \tau \neq \tau^{\prime}, \quad c_{2}(X) Z_{\tau}=28 .
$$

This raises a question: How do we interpret these geometrically?

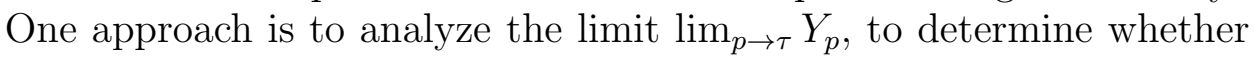
it contains $W_{\tau}$ as an irreducible component, with residual intersection in the summand $\mathbf{1}_{X}^{81}$.

The approach we take is to analyze the action of $G_{3}$ on $K_{2}(A)$ and its deformations. Regard $G_{3} \subset \operatorname{Sp}(A[3]) \ltimes A[3]$, the canonical semidirect product associated to the action of the symplectic group on $A[3]$.

Theorem 4.4 (Hodge conjecture for canonical classes). Consider the action of $G_{3}$ on $K_{2}(A)$. For each $\tau \in A[3] \subset G_{3}$, let $\iota_{\tau} \in G_{3}$ denote the involution fixing $\tau$.

(1) The fixed point locus of $\iota_{\tau}$ has two irreducible components. First, there is an isolated point corresponding to the vertex of $W_{\tau}$. Second, there is a Kummer surface that is an irreducible component of $\lim _{p \rightarrow \tau} Y_{p}$ and has class $Z_{\tau}$. For instance, we have

$$
Z_{0}=\overline{\left\{\left(a_{1}, a_{2}, a_{3}\right): a_{1}=0, a_{2}=-a_{3}, a_{2} \neq 0\right\}}
$$

and the other $Z_{\tau}$ are translates of $Z_{0}$ via the action of $A[3]$.

(2) For each deformation $X$ of $K_{2}(A)$, the $Z_{\tau}$ deforms to a submanifold of $X$.

Proof. Each $\iota_{\tau}$ is conjugate to the involution induced by the sign involution on $A$; its fixed points are clearly the stratum $Z_{0}$ and the vertex of the exceptional divisor $W_{0}$.

Proposition 3.1 shows each $\iota_{\tau}$ carries over to deformations of $X$; thus the fixed-point loci carry over as well.

Definition 4.5. Let $X$ be an irreducible holomorphic symplectic manifold, with its natural hyperkähler structure. A submanifold $Z \subset X$ is trianalytic if $Z$ is analytic with respect to each of the associated complex structures. 
These have been studied systematically by Verbitsky; see, for example, [30, 32]. One general result is that analytic subvarieties representing canonical Hodge classes are automatically trianalytic [30, Thm. 4.1]. In particular, all analytic subvarieties of (Mumford-Tate) general irreducible holomorphic symplectic manifolds have this property. The deformations of the $Z_{\tau}$ in $X$ are thus examples of trianalytic submanifolds. We lack a clear picture of what these generic deformations look like. However, their middle cohomology should have a piece that is isogenous to $\mathrm{H}^{2}(X)$. For more discussion of trianalytic subvarieties of generalized Kummer varieties, see [18, §6].

\section{The lattice of CANOnical Classes}

Proposition 5.1. The lattice $\Pi=\left\langle Z_{\tau}: \tau \in A[3]\right\rangle$ under the intersection form is positive definite of discriminant $2^{2} \cdot 3^{81} \cdot 7$. We have

$$
c_{2}(X)=\frac{1}{3} \sum_{\tau \in A[3]} Z_{\tau},
$$

which is non-divisible.

Proof. From Equation (4), we see that the intersection form of $\Pi$ is

\begin{tabular}{c|cccc} 
& $Z_{\tau_{1}}$ & $Z_{\tau_{2}}$ & $Z_{\tau_{3}}$ & $\cdots$ \\
\hline$Z_{\tau_{1}}$ & 4 & 1 & 1 & $\cdots$ \\
$Z_{\tau_{2}}$ & 1 & 4 & 1 & $\cdots$ \\
$Z_{\tau_{3}}$ & 1 & 1 & 4 & $\ddots$ \\
$\vdots$ & $\vdots$ & $\vdots$ & $\ddots$ & $\ddots$
\end{tabular}

so the corresponding matrix has eigenvalues 84 (with multiplicity one) and 3 (with multiplicity eighty).

The non-divisibility follows from

$$
c_{2}(X) \cdot Z_{j}=28, \quad c_{2}(X)^{2}=756, \quad c_{2}(X) \cdot \mathbb{P}^{2}=-3,
$$

where $\mathbb{P}^{2} \subset X$ is a Lagrangian plane.

Proposition 5.2. Consider the generalized Kummer varieties deformation equivalent to $K_{2}(A)$. The image of the monodromy representation on

$$
\Pi=\left\langle Z_{\tau}: \tau \in A[3]\right\rangle \subset \mathrm{H}^{4}(X, \mathbb{Z})
$$

contains the semidirect product

$$
\operatorname{Sp}(A[3]) \ltimes A[3],
$$

where $\operatorname{Sp}(A[3])$ is the symplectic group. These groups act on the $Z_{\tau}, \tau \in$ $A[3]$ via permutation. 
Proof. The monodromy representation for abelian surfaces acts on their three-torsion via the symplectic group $\operatorname{Sp}(A[3])$; this group acts on $\Pi$ as well via the permutation representation on the $Z_{\tau}$. In addition, translation by a three-torsion element $\tau^{\prime} \in A[3]$ induces a non-trivial action on $K_{2}(A)$; the action on $\Pi$ corresponds to the permutation

$$
Z_{\tau} \mapsto Z_{\tau+\tau^{\prime}}
$$

\section{Comparison Between Hilbert schemes and Generalized KUMMER VARIETIES}

The middle cohomology of the Hilbert scheme $Y$ of length-two subschemes of a K3 surface is generated by the second cohomology. Thus the class of a Lagrangian plane in $Y$ can be written as a quadratic polynomial in $\mathrm{H}^{2}(Y)$. After suitable deformation, it can be written as a linear combination of $c_{2}(Y)$ and $\lambda^{2}$, where $\lambda$ is proportional to the class of a line. This Ansatz allowed us to compute $(\lambda, \lambda)$ in this case [15, §4].

In our situation, the presence of 80 additional classes complicates the algebra. The following proposition shows these must be included in any formula for the class of a Lagrangian plane:

Proposition 6.1. Let $X$ be deformation equivalent to $K_{2}(A)$ and $P \subset$ $X$ a Lagrangian plane. Let $\lambda \in \mathrm{H}^{2}(X, \mathbb{Z})$ be $6[\ell]$ where $\ell \subset P$ is a line. Then we cannot write

$$
[P]=a \lambda^{2}+b c_{2}(X)
$$

for any rational $a, b \in \mathbb{Q}$.

Proof. Recall that [29, p. 123]

$$
c_{2}(X)^{2}=756
$$

and [6, §5],[5, Thm. 6]

$$
\chi\left(\mathcal{O}_{X}(L)\right)=3\left(\begin{array}{c}
\frac{(L, L)}{2}+2 \\
2
\end{array}\right)=L^{4} / 4 !+L^{2} c_{2}(X) / 24+\chi\left(\mathcal{O}_{X}\right) .
$$

Thus we find

$$
L^{4}=9(L, L)^{2}, L^{2} c_{2}(X)=54(L, L) .
$$

The condition $[P]^{2}=3$ translates into

$$
3=9 a^{2}(\lambda, \lambda)^{2}+108 a b(\lambda, \lambda)+756 b^{2} .
$$

The condition $c_{2}(X) \mid \mathbb{P}^{2}=-3$ yields

$$
-3=54 a(\lambda, \lambda)+756 b .
$$


Finally, the fact that $\lambda \mid \mathbb{P}^{2}=(\lambda, \ell) \ell$ implies

$$
(\lambda, \lambda)^{2} / 36=9 a(\lambda, \lambda)^{2}+54 b(\lambda, \lambda) .
$$

These equations admit no solutions for $a, b,(\lambda, \lambda) \in \mathbb{Q}$.

\section{A KEY SPECIAL CASE}

In order to formulate a revised Ansatz, we analyze a specific example: Let $A=E_{1} \times E_{2}$ and consider the planes $P \subset K_{2}(A)$ associated with the linear series $3 \cdot(0)$ in $E_{1} \times 0$. Let $\Lambda^{\prime}=E_{1}[3] \times 0 \subset A[3], \ell$ the class of a line in $P$, and

$$
\lambda=6 \ell=6 E_{1}-3 e
$$

the corresponding class in $\mathrm{H}^{2}\left(K_{2}(A), \mathbb{Z}\right)$.

\section{Proposition 7.1.}

$$
[P]=\frac{1}{216} \lambda^{2}+\frac{1}{8} c_{2}\left(K_{2}(A)\right)-\frac{1}{3} \sum_{\tau \in \Lambda^{\prime}} Z_{\tau}
$$

Proof. We start with the Ansatz

$$
[P]=a \lambda^{2}+b c_{2}(X)+\widehat{Z}
$$

where $\widehat{Z} \in c_{2}(X)^{\perp} \cap\left\langle Z_{\tau}: \tau \in A[3]\right\rangle$.

Recall that [29, p. 123]

$$
c_{2}(X)^{2}=756
$$

and [6, §5],[5, Thm. 6]

$$
\chi\left(\mathcal{O}_{X}(L)\right)=3\left(\begin{array}{c}
\frac{(L, L)}{2}+2 \\
2
\end{array}\right)=L^{4} / 4 !+L^{2} c_{2}(X) / 24+\chi\left(\mathcal{O}_{X}\right) .
$$

Thus we find

$$
L^{4}=9(L, L)^{2}, \quad L^{2} c_{2}(X)=54(L, L) .
$$

Furthermore, we have

$$
L_{1} L_{2} L_{3} L_{4}=3\left[\left(L_{1}, L_{2}\right)\left(L_{3}, L_{4}\right)+\left(L_{1}, L_{3}\right)\left(L_{2}, L_{4}\right)+\left(L_{1}, L_{4}\right)\left(L_{2}, L_{3}\right)\right] \text {. }
$$

The condition $[P]^{2}=3$ translates into

$$
3=9 a^{2}(\lambda, \lambda)^{2}+108 a b(\lambda, \lambda)+756 b^{2}+\widehat{Z} \cdot \widehat{Z} .
$$

The condition $c_{2}(X) \mid \mathbb{P}^{2}=-3$ yields

$$
-3=54 a(\lambda, \lambda)+756 b .
$$

Finally, the fact that $\lambda \mid \mathbb{P}^{2}=(\lambda, \ell) \ell$ implies

$$
(\lambda, \lambda)^{2} / 36=9 a(\lambda, \lambda)^{2}+54 b(\lambda, \lambda) .
$$


Given that $(\lambda, \lambda)=-54$, the last two equations allow us to solve for $a$ and $b$, i.e., we find $a=1 / 216$ and $b=1 / 72$. Thus we have

$$
[P]=\frac{1}{216} \lambda^{2}+\frac{1}{72} c_{2}\left(K_{2}(A)\right)+\widehat{Z} .
$$

It follows that

$$
\begin{aligned}
{[P] \cdot e^{2} } & =\frac{1}{216} \lambda^{2} e^{2}+\frac{54}{72}(e, e) \\
& =\frac{3}{216}\left((\lambda, \lambda)(e, e)+2(\lambda, e)^{2}\right)-9 / 2 \\
& =9 .
\end{aligned}
$$

We apply the formulas from Section 4, We know that

$$
P \cdot[W]=P \cdot \frac{3}{8}\left(c_{2}(X)+3 e^{2}\right)=-9 / 8+81 / 8=9
$$

and

$$
P \cdot\left[Y_{p}\right]=P \cdot \frac{1}{72}\left(3 c_{2}(X)+e^{2}\right)=0 .
$$

The geometry of $P$ shows it is disjoint from $W_{\tau}$ for $\tau \notin \Lambda^{\prime}$. By symmetry, we conclude that

$$
P \cdot\left[W_{\tau}\right]=1, \quad \tau \in \Lambda^{\prime} .
$$

It follows that

$$
P \cdot Z_{\tau}=\left\{\begin{array}{l}
0 \text { if } \tau \notin \Lambda^{\prime} \\
-1 \text { if } \tau \in \Lambda^{\prime},
\end{array}\right.
$$

which reflects the fact that $P$ fails to intersect these trianalytic varieties transversally.

Thus for $\tau \in \Lambda^{\prime}$ we have

$$
\begin{aligned}
\widehat{Z} \cdot Z_{\tau} & =\left([P]-\frac{1}{216} \lambda^{2}-\frac{1}{72} c_{2}(X)\right) \cdot Z_{\tau} \\
& =-1-\frac{1}{216} 2(\lambda, \lambda)-\frac{1}{72} 28 \\
& =-8 / 9 .
\end{aligned}
$$

Since the $\tau \in \Lambda^{\prime}$ appear symmetrically in $\widehat{Z}$, we conclude that

$$
\widehat{Z}=\alpha\left(\frac{1}{3} c_{2}(X)-\sum_{\tau \in \Lambda^{\prime}} Z_{\tau}\right) .
$$

We have seen that

$$
Z_{\tau} Z_{\tau^{\prime}}= \begin{cases}4 & \text { if } \tau=\tau^{\prime} \\ 1 & \text { if } \tau \neq \tau^{\prime}\end{cases}
$$

thus $\alpha=1 / 3$ and we conclude

$$
[P]=\frac{1}{216} \lambda^{2}+\frac{1}{72} c_{2}(X)+\frac{1}{3}\left(c_{2}(X) / 3-\sum_{\tau \in \Lambda^{\prime}} Z_{\tau}\right),
$$

which yields the desired formula. 


\section{INTEGRALITY}

The example in Section 7 allows us to obtain further integrality results:

Proposition 8.1. Let $\Lambda^{\prime} \subset A[3]$ be a translate of a non-isotropic twodimensional subspace. Then

$$
\frac{1}{8} c_{2}(X)-\frac{1}{3} \sum_{\tau \in \Lambda^{\prime}} Z_{\tau}
$$

intersects each class of $\Pi$ integrally.

This statement follows from the computation in Proposition 7.1 and Proposition 5.2, which implies that the monodromy acts transitively on translates of non-isotropic subspaces. These are precisely the projections of planes described above (and their orbits under the monodromy action of $\operatorname{Sp}(A[3])$ and translation by $A[3])$ into $\Pi$.

Proposition 8.2. Let $\Pi^{\prime}$ denote the dual of the lattice $\Pi$, with the $\mathbb{Q}$ valued intersection form induced by $\Pi^{\prime} \subset \Pi \otimes \mathbb{Q}$. Then $\Pi^{\prime}$ is generated by

- $\frac{1}{28} c_{2}(X)=\frac{1}{84} \sum_{\tau} Z_{\tau}$;

- $\frac{1}{3} \sum_{\tau} \beta_{\tau} Z_{\tau}$, where $\beta_{\tau} \in \mathbb{Z}$ and satisfy $\sum_{\tau} \beta_{\tau} \equiv 0(\bmod 3)$.

Proof. It is evident that these classes generate a subgroup containing $\Pi$ and intersect each of the $Z_{\tau}$ integrally. Thus if $M$ is the lattice they generate, we have

$$
\Pi \subset M \subset \Pi^{\prime} \text {. }
$$

We compute the index of $\Pi$ in $M$. The sublattice $M_{2} \subset \Pi^{\prime}$ generated by the classes $\frac{1}{3} \sum_{\tau} \beta_{\tau} Z_{\tau}$ as above factors

$$
\Pi \subset M_{2} \subset \frac{1}{3} \Pi \text {. }
$$

The image of $M_{2}$ in $\frac{1}{3} \Pi / \Pi \simeq(\mathbb{Z} / 3 \mathbb{Z})^{81}$ is a hyperplane, thus $\Pi \subset M_{2}$ has index $3^{80}$. Note that $c_{2}(X) \in M_{2}$ as a primitive vector, thus

$$
M_{2} \subset M_{2}+\frac{1}{28} c_{2}(X)=M
$$

has index 28. Thus the index of $\Pi \subset M$ is $3^{80} \cdot 28$, which is the discriminant.

Proposition 8.3. Consider the vectors $Z_{\tau}^{\prime}=\frac{1}{3}\left(\frac{c_{2}(X)}{28}-Z_{\tau}\right)$ for each $\tau$, which have intersections

$$
Z_{\tau_{1}}^{\prime} Z_{\tau_{2}}^{\prime}=\left\{\begin{array}{ll}
\frac{83}{28 \cdot 9} & \text { if } \tau_{1}=\tau_{2} \\
\frac{-1}{28.9} & \text { if } \tau_{1} \neq \tau_{2},
\end{array} .\right.
$$


We have

$$
\Pi^{\prime}=\left\{\sum_{\tau} \beta_{\tau} Z_{\tau}^{\prime}: \beta_{\tau} \in \mathbb{Z}, \sum_{\tau} \beta_{\tau} \equiv 0 \quad(\bmod 3)\right\} .
$$

The intersection form on $\Pi^{\prime}$ takes values in $\frac{1}{28 \cdot 9} \mathbb{Z}$; squares of elements are in $\frac{1}{28 \cdot 3} \mathbb{Z}$.

Proof. Only the last statement requires verification: It suffices to observe that the matrix

$$
\Psi=\left(\begin{array}{cccc}
83 & -1 & -1 & \cdots \\
-1 & 83 & -1 & \ddots \\
-1 & -1 & \ddots & \vdots \\
\vdots & \ddots & \cdots & 83
\end{array}\right)
$$

is congruent to

$$
\left(\begin{array}{cccc}
-1 & -1 & -1 & \cdots \\
-1 & -1 & -1 & \ddots \\
-1 & -1 & \ddots & \vdots \\
\vdots & \ddots & \cdots & -1
\end{array}\right)
$$

modulo three. Elements summing to zero modulo three are in the kernel.

\section{Computing $(\lambda, \lambda)$}

We formulate a new Ansatz in light of the example in Section 7.

Let $P$ be a class of a plane, which we now assume can be written

$$
P=a \lambda^{2}+b c_{2}(X)+\widehat{Z}
$$

as above and in the proof of Proposition 7.1. Note that $\widehat{Z}^{2} \geq 0$ as the lattice $\Pi$ is positive definite, thus

$$
\left(a \lambda^{2}+b c_{2}(X)\right)^{2} \leq 3
$$

Using the equations

$$
\begin{aligned}
-3 & =54 a(\lambda, \lambda)+b 756 \\
\frac{(\lambda, \lambda)^{2}}{36} & =9 a(\lambda, \lambda)^{2}+54 b(\lambda, \lambda)
\end{aligned}
$$

to eliminate $a$ and $b$, we find

$$
\left(a \lambda^{2}+b c_{2}(X)\right)^{2}=\frac{1}{46656}\left(7(\lambda, \lambda)^{2}+108(\lambda, \lambda)+972\right) .
$$

This is always positive, and is $\leq 3$ only when $|(\lambda, \lambda)| \leq 150$. Thus there are only finitely many possibilities for $(\lambda, \lambda)$. 
We introduce a new class: Let $\mu \in \mathbb{Z} \lambda^{2}+\mathbb{Z} c_{2}(X)$ denote a primitive class orthogonal to $c_{2}(X)$ such that $\lambda^{2}$ appears with a positive coefficient. This is unique up to a positive scalar; we'll choose a specific representative in the next lemma. Note that $\mu$ is also orthogonal to each of the $Z_{\tau}$ and $Z_{\tau}^{\prime}$, by Proposition 4.1.

Lemma 9.1. We have $\mu^{2}>0$.

Proof. We may write

$$
\mu=7 \lambda^{2}-\frac{(\lambda, \lambda)}{2} c_{2}(X)
$$

which is orthogonal to $c_{2}(X)$. Using the intersection numbers computed above, we find

$$
\mu^{2}=49 \cdot 9(\lambda, \lambda)^{2}-7 \cdot 54(\lambda, \lambda)^{2}+756 / 4(\lambda, \lambda)^{2}=252(\lambda, \lambda)^{2} .
$$

We write the class of a Lagrangian plane in terms of this orthogonal decomposition; since $[P]$ is integral, its projection onto each summand lies in the dual to the underlying lattice. In particular, we have

$$
[P]=\alpha \mu+\sum_{\tau} \beta_{\tau} Z_{\tau}^{\prime}, \beta_{\tau} \in \mathbb{Z}, \alpha \in \mathbb{Q},
$$

and $\sum_{\tau} \beta_{\tau} \equiv 0(\bmod 3)$. If $a$ is the constant in Equation (7) we have $a=7 \alpha$. Combining the equations above with these, we find

$$
(\alpha \mu)^{2}=a^{2}(\lambda, \lambda)^{2} \frac{36}{7} \text {. }
$$

Write

$$
S=\left(\sum_{\tau} \beta_{\tau} Z_{\tau}^{\prime}\right)^{2}=P^{2}-(\alpha \mu)^{2}=3-a^{2}(\lambda, \lambda)^{2} \frac{36}{7}
$$

and use the equations above to eliminate $a$. We find

$$
\begin{aligned}
S & =\frac{-49(\lambda, \lambda)^{2}-756(\lambda, \lambda)+976860}{326592} \\
& =\frac{-49(\lambda, \lambda)^{2}-756(\lambda, \lambda)+2^{2} \cdot 3^{6} \cdot 5 \cdot 67}{2^{6} \cdot 3^{6} \cdot 7}
\end{aligned}
$$

The lattice $\left\langle Z_{\tau}^{\prime}\right\rangle$ is positive definite, so $S>0$. This implies

$$
-150 \leq(\lambda, \lambda)<134 \text {. }
$$


We know that $9 \cdot 28 \cdot S$ is an integer represented by the quadratic form (6), and thus is divisible by 3 . We therefore obtain the following divisibility

$$
2^{4} 3^{5} \mid 49(\lambda, \lambda)^{2}+756(\lambda, \lambda)-2^{2} \cdot 3^{6} \cdot 5 \cdot 67 .
$$

Thus we find

$$
(\lambda, \lambda) \equiv 2 \quad(\bmod 4), \quad(\lambda, \lambda) \equiv 0 \quad(\bmod 27) .
$$

The only admissible values in the range $(-150,134)$ are

$$
(\lambda, \lambda)= \pm 54
$$

It follows that

$$
S=\left(\sum_{\tau} \beta_{\tau} Z_{\tau}^{\prime}\right)^{2}=75 / 28
$$

and $9 \cdot 75=675$ is represented by (마 $)$.

\section{AnAlysis of SHORT VECTORS}

We complete the proof of Theorem 1.1 and extract additional information on how classes of Lagrangian planes project onto the tautological classes.

Lemma 10.1. Let $\sum_{\tau} \beta_{\tau} Z_{\tau}^{\prime}$ denote the projection of $P$ onto the sublattice $\Pi^{\prime}$, as described in (8)). Then we have $\sum_{\tau} \beta_{\tau}=9$.

Proof. Recall that $c_{2}(X)[P]=-3$ and $c_{2}(X) \mu=0$, so that

$$
c_{2}(X)\left(\sum_{\tau} \beta_{\tau} Z_{\tau}^{\prime}\right)=-3
$$

On the other hand, we have

$$
c_{2}(X) Z_{\tau}^{\prime}=c_{2}(X)\left(c_{2}(X) / 84-1 / 3 Z_{\tau}\right)=-1 / 3
$$

so we find

$$
c_{2}(X)\left(\beta_{\tau} Z_{\tau}^{\prime}\right)=-1 / 3 \sum_{\tau} \beta_{\tau}
$$

The equation follows.

Let $\Psi=9 \cdot 28 \cdot \Pi^{\prime}$, i.e., the dual to $\Pi$ renormalized to an integral form as in (6) . Then the Lemma implies

$$
\begin{aligned}
\left(\sum_{\tau} \beta_{\tau} Z_{\tau}\right)_{\Psi}^{2}= & 84\left(\sum_{\tau} \beta_{\tau}^{2}\right)-\left(\sum_{\tau} \beta_{\tau}\right)^{2} \\
& 84\left(\sum_{\tau} \beta_{\tau}^{2}\right)-81
\end{aligned}
$$


This means that $9 \cdot 28 \cdot S-81 \equiv 0(\bmod 84)$, which yields the congruences

$$
-49(\lambda, \lambda)^{2}-756(\lambda, \lambda)+2^{2} \cdot 3^{6} \cdot 7 \cdot 51 \equiv 0 \quad\left(\bmod 2^{6} \cdot 3^{5} \cdot 7\right),
$$

which are stronger than those in (10). We obtain that

$$
\lambda \equiv 2 \quad(\bmod 8), \quad \lambda \equiv 0 \quad(\bmod 27) .
$$

Combining this with the bounds (9), we conclude that

$$
(\lambda, \lambda)=-54 \text {. }
$$

Since $\lambda=6 \ell$, we deduce that $(\ell, \ell)=-3 / 2$, which completes the proof of Theorem 1.1 .

Proposition 10.2. We have $\beta_{\tau}=0$ for all but nine values of $\tau$, for which $\beta_{\tau}=1$.

Proof. We have shown (see (11) above) that $S=75 / 28$, which means that

$$
84 \sum_{\tau} \beta_{\tau}^{2}=81+9 \cdot 28 \cdot S=756
$$

whence

$$
\sum_{\tau} \beta_{\tau}^{2}=\sum_{\tau} \beta_{\tau}=9
$$

Since the $\beta_{\tau}$ are integers, at most 9 of them are nonzero, and we may as well restrict to that subspace.

By basic calculus, the maximal value of the function $x_{1}+\cdots+x_{n}$ on the unit sphere is $\sqrt{n}$, achieved precisely at $x_{1}=x_{2}=\cdots=x_{n}=$ $1 / \sqrt{n}$. Applying this to our nine-dimensional subspace gives the result.

\section{Monodromy AND INTERSECTION ANALYSIS}

We continue to identify the set of all $\tau$ with $A[3]$, the three-torsion of an abelian surface. The following Proposition summarizes what we have obtained so far:

Proposition 11.1. Let $X$ be deformation equivalent to $K_{2}(A), P \subset X$ a Lagrangian plane, $\ell \in \mathrm{H}_{2}(X, \mathbb{Z})$ the class of a line on the plane, and $\lambda=6 \ell \in \mathrm{H}^{2}(X, \mathbb{Z})$. Then we have

$$
\begin{aligned}
{[P] } & =\frac{1}{216} \lambda^{2}+\frac{1}{56} c_{2}(X)+\sum_{\tau \in \Lambda} Z_{\tau}^{\prime} \\
& =\frac{1}{216} \lambda^{2}+\frac{1}{8} c_{2}(X)-\frac{1}{3} \sum_{\tau \in \Lambda} Z_{\tau},
\end{aligned}
$$

where $Z_{\tau}^{\prime}=\frac{1}{3}\left(\frac{c_{2}(X)}{28}-Z_{\tau}\right)$ and $\Lambda \subset A[3]$ is a set of cardinality nine. 
We recall the following geometric facts:

- the group $G_{3}$ acts on $X$, and thus on $\left\{Z_{\tau}: \tau \in A[3]\right\}$ and $\Pi$, as described in Proposition 3.1,

- the $Z_{\tau}$ are canonical trianalytic submanifolds, and they deform as $X$ deforms (see the end of Section 4);

- the monodromy group acts on $\left\{Z_{\tau}\right\}$ and $\Pi$, as described in Proposition 5.2 , given a distinguished base point $\tau_{0}$, the intersection of the monodromy group with the stabilizer of $\tau_{0}$ contains the symplectic group $\operatorname{Sp}(A[3])$, where $\tau_{0}$ is interpreted as 0 .

Theorem 11.2. Suppose $P \subset X$ is a Lagrangian plane as in Proposition [11.1. Then $\Lambda \subset A[3]$ is a translate of a two-dimensional subspace.

Note that the example presented in Section 7 and the facts on monodromy/automorphisms quoted above imply that every translate $\Lambda$ of a two-dimensional non-isotropic subspace arises from a plane in a deformation of $X$.

Problem 11.3. Can isotropic subspaces arise from Lagrangian planes?

Corollary 11.4. Suppose that $P \subset X$ is a plane contained in a manifold deformation equivalent to $K_{2}(A)$. Then the orbit of $P$ under $\operatorname{Aut}(X)$ contains nine distinct planes; the stabilizer of $P$ has order dividing nine.

Proposition 11.5. Let $\Lambda$ denote a set of points arising from a Lagrangian plane. Then

$$
\#\left\{\Lambda \cap\left(\Lambda+\tau_{0}\right)\right\} \quad(\bmod 3)
$$

is constant as $\tau_{0}$ varies.

Proof. We have

$$
\left(\sum_{\tau \in \Lambda} Z_{\tau}^{\prime}\right)^{2} \equiv\left(\sum_{\tau \in \Lambda} Z_{\tau}^{\prime}\right)\left(\sum_{\tau \in\left(\Lambda+\tau_{0}\right)} Z_{\tau}^{\prime}\right) \quad(\bmod \mathbb{Z}),
$$

because if $P_{2}$ is the corresponding plane then

$$
P^{2}-P \cdot P_{2} \in \mathbb{Z}
$$

which translates into the assertion of the lemma.

The corresponding vectors have pairing (with respect to $\Psi$ ) divisible by $9 \cdot 28$

$$
\left(\sum_{\tau \in \Lambda} Z_{\tau}^{\prime}\right) \cdot \Psi\left(\sum_{\tau \in\left(\Lambda+\tau_{0}\right)} Z_{\tau}^{\prime}\right) \equiv 0 \quad(\bmod 9 \cdot 28)
$$


Modulo $9 \cdot 28$ we find

$$
83 \#\left\{\tau \in \Lambda \cap\left(\Lambda+\tau_{0}\right)\right\}-\#\left\{(\tau, \widetilde{\tau}): \tau \neq \widetilde{\tau}, \tau \in \Lambda, \widetilde{\tau} \in\left(\Lambda+\tau_{0}\right)\right\} \equiv 0
$$

and

$$
84 \#\left\{\tau \in \Lambda \cap\left(\Lambda+\tau_{0}\right)\right\}-\#\left\{(\tau, \widetilde{\tau}): \tau \in \Lambda, \widetilde{\tau} \in\left(\Lambda+\tau_{0}\right)\right\} \equiv 0 .
$$

Since $|\Lambda|=\left|\Lambda+\tau_{0}\right|=9$, we have

$$
84 \#\left\{\tau \in \Lambda \cap\left(\Lambda+\tau_{0}\right)\right\} \equiv 81 \quad(\bmod 9 \cdot 28)
$$

and

$$
\#\left\{\tau \in \Lambda \cap\left(\Lambda+\tau_{0}\right)\right\} \equiv 0 \quad(\bmod 3) .
$$

Remark 11.6. This is insufficient to characterize translates of twodimensional subspaces in $A[3] \simeq(\mathbb{Z} / 3 \mathbb{Z})^{4}$. For instance, consider the set

$$
\left\{e_{2}, e_{2}+e_{1}, e_{2}-e_{1}, e_{3}, e_{3}+e_{1}, e_{3}-e_{1}, e_{4}, e_{4}+e_{1}, e_{4}-e_{1}\right\} .
$$

Every element of the orbit of this set under $G_{3}$ meets the set in 0 or 3 points. Thus our intersection condition is insufficient to establish Theorem 11.2.

We strengthen the analysis above. Suppose $P$ and $\widetilde{P}$ are planes of the form

$$
[P]=\alpha \mu+\sum_{\tau} \beta_{\tau} Z_{\tau}^{\prime}, \quad[\widetilde{P}]=\alpha \mu+\sum_{\tau} \widetilde{\beta}_{\tau} Z_{\tau}^{\prime},
$$

so the difference is an integral class

$$
[P]-[\widetilde{P}]=\sum_{\tau}\left(\beta_{\tau}-\widetilde{\beta}_{\tau}\right) Z_{\tau}^{\prime}
$$

The nonzero coefficients are all \pm 1 . Applying the symplectic group $\operatorname{Sp}(A[3])$, we get additional integral classes. In particular, the classes

$$
\sum_{\tau \in \widetilde{\Lambda}} Z_{\tau}^{\prime}-Z_{\tau+\tau_{0}}^{\prime}
$$

are integral for each non-isotropic $\widetilde{\Lambda} \subset A[3]$ and $\tau_{0} \in A[3]$.

Proposition 11.7. Let $\widetilde{\Lambda}$ denote a non-isotropic subspace and $\Lambda$ a set of points arising from a Lagrangian plane. Then

$$
\#\left\{\Lambda \cap\left(\widetilde{\Lambda}+\tau_{0}\right)\right\} \quad(\bmod 3)
$$

is constant as $\tau_{0}$ varies. 
Proof. Consider the classes $\sum_{\tau \in \Lambda} Z_{\tau}^{\prime}, \sum_{\tau \in \widetilde{\Lambda}} Z_{\tau}^{\prime}$, and $\sum_{\tau \in \Lambda+\tau_{0}} Z_{\tau}^{\prime}$. We have

$$
\left(\sum_{\tau \in \Lambda} Z_{\tau}^{\prime}\right) \cdot\left(\sum_{\tau \in \widetilde{\Lambda}+\tau_{0}} Z_{\tau}^{\prime}-\sum_{\tau \in \widetilde{\Lambda}} Z_{\tau}^{\prime}\right) \in \mathbb{Z}
$$

which translates into

$$
\left(\sum_{\tau \in \Lambda} Z_{\tau}^{\prime}\right) \cdot \Psi\left(\sum_{\tau \in \widetilde{\Lambda}+\tau_{0}} Z_{\tau}^{\prime}-\sum_{\tau \in \widetilde{\Lambda}} Z_{\tau}^{\prime}\right) \equiv 0 \quad(\bmod 9 \cdot 28) .
$$

Thus we have

$$
\begin{aligned}
& 83 \#\left\{\tau \in \Lambda \cap\left(\widetilde{\Lambda}+\tau_{0}\right)\right\}-\#\left\{(\tau, \widetilde{\tau}): \tau \neq \widetilde{\tau}, \tau \in \Lambda, \widetilde{\tau} \in \widetilde{\Lambda}+\tau_{0}\right\} \\
& \equiv 83 \#\{\tau \in \Lambda \cap \widetilde{\Lambda}\}-\#\{(\tau, \widetilde{\tau}): \tau \neq \widetilde{\tau}, \tau \in \Lambda, \widetilde{\tau} \in \widetilde{\Lambda}\} \quad(\bmod 9 \cdot 28)
\end{aligned}
$$

and

$$
\begin{aligned}
& 84 \#\left\{\tau \in \Lambda \cap\left(\widetilde{\Lambda}+\tau_{0}\right)\right\}-\#\left\{(\tau, \widetilde{\tau}): \tau \in \Lambda, \widetilde{\tau} \in\left(\widetilde{\Lambda}+\tau_{0}\right)\right\} \\
& \equiv 84 \#\{\tau \in \Lambda \cap \widetilde{\Lambda}\}-\#\{(\tau, \widetilde{\tau}): \tau \in \Lambda, \widetilde{\tau} \in \widetilde{\Lambda}\} \quad(\bmod 9 \cdot 28) .
\end{aligned}
$$

Since $|\Lambda|=|\widetilde{\Lambda}|=\left|\widetilde{\Lambda}+\tau_{0}\right|=9$, we obtain

$$
\#\left\{\tau \in \Lambda \cap\left(\widetilde{\Lambda}+\tau_{0}\right)\right\} \equiv \#\{\tau \in \Lambda \cap \widetilde{\Lambda}\} \quad(\bmod 3),
$$

which is what we sought to prove.

We shall need the following result on finite geometries, which should be understood in the context of Radon transforms over finite fields [35]:

Proposition 11.8. Let $V$ be a four-dimensional vector space over a finite field with $q$ elements, with $q$ odd. Suppose that $V$ admits a symplectic form. Suppose that $\Lambda \subset V$ is a subset with $q^{2}$ elements such that, for each affine non-isotropic plane $\widetilde{\Lambda} \subset V$, the function

$$
\begin{aligned}
V & \rightarrow \mathbb{Z} / q \mathbb{Z} \\
\tau & \mapsto \#\{\Lambda \cap(\widetilde{\Lambda}+\tau)\} \quad(\bmod q)
\end{aligned}
$$

is constant. Then $\Lambda$ is an affine plane in $V$.

Proof. It suffices to show that $\Lambda$ is 'convex', in the sense that for any pair of distinct $\tau_{1}, \tau_{2} \in \Lambda$, the affine line $\mathfrak{l}\left(\tau_{1}, \tau_{2}\right)$ is contained in $\Lambda$. Suppose this is not the case, so in particular

$$
\#\left\{\mathfrak{l}\left(\tau_{1}, \tau_{2}\right) \cap \Lambda\right\}<q .
$$

For simplicity, assume that $\tau_{1}=0$ so that every affine plane containing $\mathfrak{l}\left(\tau_{1}, \tau_{2}\right)$ is a subspace. Let $\operatorname{Gr}(2, V)$ denote the Grassmannian (a smooth quadric hypersurface in $\left.\mathbb{P}^{5}\right), \operatorname{IGr}(2, V)$ the isotropic Grassmannian (a smooth hyperplane section of $\operatorname{Gr}(2, V)$ ), and $\Sigma \subset \operatorname{Gr}(2, V)$ the Schubert variety of planes containing $\mathfrak{l}\left(\tau_{1}, \tau_{2}\right)$ (which is isomorphic 
to $\left.\mathbb{P}^{2}\right)$. Note that $\Sigma \not \subset \operatorname{IGr}(2, V)$, since the latter is a smooth quadric threefold. Thus

$$
\Sigma_{\circ}:=\Sigma \cap(\operatorname{Gr}(2, V) \backslash \operatorname{IGr}(2, V)) \simeq \mathbb{A}^{2}
$$

which has $q^{2}$ points over our finite field.

The planes parametrized by $\Sigma_{\circ}$ are disjoint away from $\mathfrak{l}\left(\tau_{1}, \tau_{2}\right)$. Hence the pigeon-hole (Dirichlet) principle guarantees there exists at least one such plane $\widetilde{\Lambda}$ that contains no points of $\Lambda$ outside $\mathfrak{l}\left(\tau_{1}, \tau_{2}\right)$. Otherwise, $\Lambda$ would have more than $q^{2}$ points. Consequently,

$$
m:=\#\{\Lambda \cap \widetilde{\Lambda}\}=\#\left\{\Lambda \cap \mathfrak{l}\left(\tau_{1}, \tau_{2}\right)\right\}
$$

is between 2 and $q-1$.

Consider the affine translates $\widetilde{\Lambda}+\tau$, for $\tau$ taken from a set of coset representatives of $V / \widetilde{\Lambda}$. These are $q^{2}$ disjoint affine planes, each with at least $m$ points of $\Lambda$. Thus $\Lambda$ has cardinality at least $m q^{2}>q^{2}$, a contradiction.

\section{FURTHER REMARKS ON SATURATION}

Problem 12.1. Characterize the saturation of $\Pi$, i.e., the intersection

$$
\Pi^{\text {sat }}=(\Pi \otimes \mathbb{Q}) \cap \mathrm{H}^{4}(X, \mathbb{Z}) .
$$

Example 12.2. Let $S=K_{1}(A)$ be a Kummer surface, $Z_{\tau}$ the (-2)classes associated with $A[2]$, and $\Pi=\left\langle Z_{\tau}: \tau \in A[2]\right\rangle$ which has discriminant $2^{16}$. The saturation of $\Pi$ in $\mathrm{H}^{2}(S, \mathbb{Z})$ is computed in [21, §3]; an element

$$
\frac{1}{2} \sum_{\tau} \epsilon(\tau) Z_{\tau}, \quad \epsilon(\tau)=0,1 \in \mathrm{H}^{2}(S, \mathbb{Z})
$$

if and only if $\epsilon: A[2] \rightarrow \mathbb{Z} / 2 \mathbb{Z}$ is affine linear. In particular, the saturation has discriminant $2^{16-2 \cdot 5}=2^{6}$, since the affine linear functions have dimension five over $\mathbb{Z} / 2 \mathbb{Z}$.

We exhibit generators admitting geometric interpretations. For each $\Lambda \subset A[2]$ non-isotropic of dimension two, the class

$$
\frac{1}{2}\left(\sum_{\tau \in \Lambda} Z_{\tau}-\sum_{\tau \in\left(\Lambda+\tau_{0}\right)} Z_{\tau}\right)
$$

is integral for geometric reasons. Analogously to the example in Section 7. we can consider $A=E_{1} \times E_{2}$ and $\mathbb{P}^{1}$ 's corresponding to degreetwo linear series on the factors. Taking difference of one such $\mathbb{P}^{1}$ and its translate, we obtain classes of the form (13).

We claim that the extension of $\Pi$ corresponding to these geometric classes agrees with the extension associated with affine linear forms. 
Suppose that $\Lambda$ is non-isotropic through the origin, defined by $x=y=$ 0 ; over $\mathbb{Z} / 2 \mathbb{Z}$ it can be defined as $x^{2}+x y+y^{2}=0$. Taking the difference of this quadric and a translate, we find

$x^{2}+x y+y^{2}-(x+a)^{2}-(x+a)(y+b)-\left(y+b^{2}\right)=-x b-y a-a^{2}-a b-b^{2}$,

which span all affine linear forms involving $x$ and $y$. Varying over all such $\Lambda$, we get the space of all affine linear forms.

The same construction is applicable to $K_{2}(A)$ as well: The additional classes (12) give an extension of $\Pi=\left\langle Z_{\tau}\right\rangle$ by a subgroup isomorphic to the $\mathbb{Z} / 3 \mathbb{Z}$-vector space of affine linear forms on $A[3]$. The resulting lattice has discriminant $2^{2} \cdot 3^{71} \cdot 7$. However, this is far from the full saturation, because the discriminant of the lattice

$$
\operatorname{Sym}^{2}\left(\mathrm{H}^{2}(X, \mathbb{Z})\right) \cap c_{2}(X)^{\perp}
$$

is much smaller. Indeed, using the formula

$$
\begin{array}{r}
D_{1} D_{2} D_{3} D_{4}=3\left(\left(D_{1}, D_{2}\right)\left(D_{3}, D_{4}\right)+\left(D_{1}, D_{3}\right)\left(D_{2}, D_{4}\right)\right. \\
\left.+\left(D_{1}, D_{4}\right)\left(D_{2}, D_{3}\right)\right)
\end{array}
$$

for $D_{1}, D_{2}, D_{3}, D_{4} \in \mathrm{H}^{2}(A, \mathbb{Z}) \subset \mathrm{H}^{2}\left(K_{2}(A), \mathbb{Z}\right)$, we can show that $\operatorname{Sym}^{2}\left(\mathrm{H}^{2}\left(K_{2}(A)\right)\right)$ has discriminant $2^{14} \cdot 3^{38}$. Taking the orthogonal complement to $c_{2}(X)$ can only increase the exponent of 3 by the power of 3 appearing in $c_{2}(X)^{2}=756$.

\section{A Negative ReSUlt on isotropic subspaces}

Theorem 11.2 yields affine subspaces $\Lambda \subset A[3]$ but does specify whether they are isotropic or non-isotropic (up to translation). In Section 7, we exhibited examples of non-isotropic subspaces. Here we identify obstructions to the appearance of isotropic subspaces:

Proposition 13.1. Let $X$ and $\widetilde{X}$ denote manifolds deformation equivalent to $K_{2}(A)$. Assume there exist Lagrangian planes $P \subset X$ and $\widetilde{P} \subset \widetilde{X}$ with

$$
\begin{aligned}
& {[P]=\frac{1}{216} \lambda^{2}+\frac{1}{56} c_{2}(X)+\sum_{\tau \in \Lambda} Z_{\tau}^{\prime},} \\
& {[\widetilde{P}]=\frac{1}{216} \widetilde{\lambda}^{2}+\frac{1}{56} c_{2}(X)+\sum_{\tau \in \widetilde{\Lambda}} Z_{\tau}^{\prime},}
\end{aligned}
$$

where $\widetilde{\Lambda} \subset A[3]$ is a two-dimensional non-isotropic affine subspace. Assume $\lambda \in \mathrm{H}^{2}(X, \mathbb{Z})$ and $\widetilde{\lambda} \in \mathrm{H}^{2}(\widetilde{X}, \mathbb{Z})$ are equivalent under the monodromy action. Then $\Lambda$ is not a translate of an isotropic subspace. 
Recall that $\lambda \in \mathrm{H}^{2}(X, \mathbb{Z})$ satisfies

$$
(\lambda, \lambda)=-54, \quad\left(\lambda, \mathrm{H}^{2}(X, \mathbb{Z})\right)=6 \mathbb{Z} .
$$

Proof. Choose $\gamma$ to be an element of the monodromy group of $X$ acting on $A[3]$ via a nontrivial symplectic transformation. Note that

$$
\gamma \Lambda=\Lambda \text { or } \gamma \Lambda \cap \Lambda=\{0\}
$$

which implies

$$
\left(\sum_{\tau \in \Lambda} Z_{\tau}^{\prime}\right) \cdot\left(\sum_{\tau \in \gamma \Lambda} Z_{\tau}^{\prime}\right)=\frac{75}{28} \text { or } \frac{1}{84} .
$$

Since $P \cdot \gamma(P) \in \mathbb{Z}$ we find that

$$
\left(\frac{1}{216} \lambda^{2}+\frac{1}{56} c_{2}(X)\right) \cdot \gamma\left(\frac{1}{216} \lambda^{2}+\frac{1}{56} c_{2}(X)\right) \equiv \frac{9}{28} \text { or } \frac{-1}{84} \quad(\bmod \mathbb{Z}) .
$$

On the other hand, it is possible to produce $\gamma$ where $\gamma(\widetilde{\Lambda}) \cap \widetilde{\Lambda}$ is one-dimensional (see Proposition 5.2). Here, it is crucial that $\widetilde{\Lambda}$ be non-isotropic. Then we have

$$
\left(\sum_{\tau \in \widetilde{\Lambda}} Z_{\tau}^{\prime}\right) \cdot\left(\sum_{\tau \in \gamma \widetilde{\Lambda}} Z_{\tau}^{\prime}\right)=1-\frac{1}{28 \cdot 9}
$$

which combined with the fact that $\widetilde{P} \cdot \gamma(\widetilde{P}) \in \mathbb{Z}$ yields

$$
\left(\frac{1}{216} \widetilde{\lambda}^{2}+\frac{1}{56} c_{2}(X)\right) \cdot \gamma\left(\frac{1}{216} \widetilde{\lambda}^{2}+\frac{1}{56} c_{2}(X)\right) \equiv \frac{1}{28 \cdot 9} \quad(\bmod \mathbb{Z}) .
$$

Since $\lambda$ and $\widetilde{\lambda}$ are in the same orbit under the monodromy representation, they share common intersection properties. Thus this is incompatible with the first equation above.

\section{Divisibility PROPERTIES AND MONODROMY}

Let $X$ be deformation equivalent to $K_{n}(A)$. Note that

$$
\mathrm{H}^{2}(A, \mathbb{Z}) \simeq U^{\oplus 3}=\left(\begin{array}{ll}
0 & 1 \\
1 & 0
\end{array}\right)^{\oplus 3}
$$

because this is a unimodular lattice of signature $(3,3)$. Thus the Beauville-Bogomolov form on $\mathrm{H}^{2}(X, \mathbb{Z})$ (see (1) ) has discriminant group $d(X)=\operatorname{Hom}\left(\mathrm{H}^{2}(X, \mathbb{Z}), \mathbb{Z}\right) / \mathrm{H}^{2}(X, \mathbb{Z}) \simeq \mathbb{Z} / 2(n+1) \mathbb{Z}$

Let $\Gamma$ denote the subgroup of the orthogonal group of $\mathrm{H}^{2}(X, \mathbb{Z})$ acting trivially on $d(X)$. Consider equivalence classes of primitive vectors, i.e., primitive $D, D^{\prime} \in \mathrm{H}^{2}(X, \mathbb{Z})$ are equivalent if $(D, D)=\left(D^{\prime}, D^{\prime}\right)$,

$$
\left(D, \mathrm{H}^{2}(X, \mathbb{Z})\right)=\left(D, \mathrm{H}^{2}(X, \mathbb{Z})\right)=\langle d\rangle,
$$


and $\frac{1}{d} D=\frac{1}{d} D^{\prime}$ in $d(X)$. By [7, $\left.\S 10\right]$ (cf. [12, Lemma 3.5]) we have that $\Gamma$-orbits are equal to these equivalence classes.

In light of Markman's results on the monodromy of Hilbert schemes of K3 surfaces [23, 22], it is natural to ask the following:

Problem 14.1. Does the monodromy representation on $\mathrm{H}^{2}(X, \mathbb{Z})$ act transitively on equivalence classes of primitive vectors?

Assuming this, the class $\ell$ of a line on a Lagrangian plane would have to be in one of the following equivalence classes:

$$
\ell=E-3 e^{\vee}, 3 e^{\vee} .
$$

We expect only the primitive class occurs because

- we know of no examples where the minimal effective generator $\ell$ of an extremal ray associated to a birational contraction of holomorphic symplectic manifolds fails to be primitive;

- we conjecture [14 that classes of type $e^{\vee}$ arise from divisorial contractions, e.g., the contraction from $K_{n}(A)$ induced by the Hilbert-Chow morphism

$$
A^{[n+1]} \rightarrow A^{(n+1)} .
$$

However, these contractions are preserved under deformations that respect $e^{\vee}$ as a Hodge class.

\section{REFERENCES}

[1] S. Boissière. Chern classes of the tangent bundle on the Hilbert scheme of points on the affine plane. J. Algebraic Geom., 14(4):761-787, 2005.

[2] S. Boissière and M. Nieper-Wisskirchen. Universal formulas for characteristic classes on the Hilbert schemes of points on surfaces. J. Algebra, 315(2):924-953, 2007.

[3] S. Boissiére, M. Nieper-Wisskirchen, and A. Sarti. Higher dimensional Enriques varieties and automorphisms of generalized Kummer varieties, 2010. arXiv:1001.4728v1.

[4] J. Briançon. Description de $H$ ilb $^{n} C\{x, y\}$. Invent. Math., 41(1):45-89, 1977.

[5] M. Britze. On the Cohomology of Generalized Kummer Varieties. PhD thesis, Cologne, 2003.

[6] M. Britze and M. A. Nieper. Hirzebruch-Riemann-Roch formulae on irreducible symplectic Kähler manifolds. arXiv:math.AG/0101062.

[7] M. Eichler. Quadratische Formen und orthogonale Gruppen. Springer-Verlag, Berlin, 1974. Zweite Auflage, Die Grundlehren der mathematischen Wissenschaften, Band 63.

[8] G. Ellingsrud and S. A. Strømme. An intersection number for the punctual Hilbert scheme of a surface. Trans. Amer. Math. Soc., 350(6):2547-2552, 1998.

[9] A. Fujiki. A theorem on bimeromorphic maps of Kähler manifolds and its applications. Publ. Res. Inst. Math. Sci., 17(2):735-754, 1981. 
[10] W. Fulton. Intersection theory, volume 2 of Ergebnisse der Mathematik und ihrer Grenzgebiete. 3. Folge. A Series of Modern Surveys in Mathematics. Springer-Verlag, Berlin, second edition, 1998.

[11] L. Göttsche. Hilbert schemes of zero-dimensional subschemes of smooth varieties, volume 1572 of Lecture Notes in Mathematics. Springer-Verlag, Berlin, 1994.

[12] V. Gritsenko, K. Hulek, and G. K. Sankaran. Moduli spaces of irreducible symplectic manifolds. to appear, available at arXiv:0802.2078.

[13] B. Hassett and Y. Tschinkel. Rational curves on holomorphic symplectic fourfolds. Geom. Funct. Anal., 11(6):1201-1228, 2001.

[14] B. Hassett and Y. Tschinkel. Intersection numbers of extremal rays on holomorphic symplectic varieties, 2009. preprint, 24 pages.

[15] B. Hassett and Y. Tschinkel. Moving and ample cones of holomorphic symplectic fourfolds. Geometric and Functional Analysis, 19(4):1065-1080, 2009.

[16] D. Huybrechts. Compact hyper-Kähler manifolds: basic results. Invent. Math., 135(1):63-113, 1999.

[17] D. Huybrechts. The Kähler cone of a compact hyperkähler manifold. Math. Ann., 326(3):499-513, 2003.

[18] D. Kaledin and M. Verbistsky. Partial resolutions of Hilbert type, Dynkin diagrams and generalized Kummer varieties, 1998. arXiv:math/9812078v1.

[19] M. Kuranishi. On the locally complete families of complex analytic structures. Ann. of Math. (2), 75:536-577, 1962.

[20] E. Looijenga and V. A. Lunts. A Lie algebra attached to a projective variety. Invent. Math., 129(2):361-412, 1997.

[21] E. Looijenga and Chr. Peters. Torelli theorems for Kähler K3 surfaces. Compositio Math., 42(2):145-186, 1980/81.

[22] E. Markman. On the monodromy of moduli spaces of sheaves on $K 3$ surfaces II, 2003. arXiv:0305043v4.

[23] E. Markman. On the monodromy of moduli spaces of sheaves on $K 3$ surfaces. J. Algebraic Geom., 17(1):29-99, 2008.

[24] E. Markman. Prime exceptional divisors on holomorphic symplectic varieties and monodromy-reflections, 2009. arXiv:0912.4981v1.

[25] H. Nakajima. Heisenberg algebra and Hilbert schemes of points on projective surfaces. Ann. of Math. (2), 145(2):379-388, 1997.

[26] M. Nieper-Wisskirchen. Is $c_{2}$ a multiple of the Beauville-Bogomolov form? available at www.math.uni-augsburg.de/alg/mitarbeiter/mnieper/c2.pdf.

[27] Z. Ran. Hodge theory and deformations of maps. Compositio Math., 97(3):309$328,1995$.

[28] S. M. Salamon. On the cohomology of Kähler and hyper-Kähler manifolds. Topology, 35(1):137-155, 1996.

[29] J. Sawon. Rozansky-Witten invariants of hyperkähler manifolds. PhD thesis, Trinity College, Cambridge, 1999. arXiv:math.AG/0404360.

[30] M. Verbitsky. Tri-analytic subvarieties of hyper-Kaehler manifolds. Geom. Funct. Anal., 5(1):92-104, 1995.

[31] M. Verbitsky. Cohomology of compact hyper-Kähler manifolds and its applications. Geom. Funct. Anal., 6(4):601-611, 1996.

[32] M. Verbitsky. Trianalytic subvarieties of the Hilbert scheme of points on a $K 3$ surface. Geom. Funct. Anal., 8(4):732-782, 1998. 
[33] Cl. Voisin. Sur la stabilité des sous-variétés lagrangiennes des variétés symplectiques holomorphes. In Complex projective geometry (Trieste, 1989/Bergen, 1989), volume 179 of London Math. Soc. Lecture Note Ser., pages 294-303. Cambridge Univ. Press, Cambridge, 1992.

[34] K. Yoshioka. Moduli spaces of stable sheaves on abelian surfaces. Math. Ann., 321(4):817-884, 2001.

[35] A.V. Zelevinski. Generalized Radon transforms in spaces of functions on Grassmann manifolds over a finite field. Uspehi Mat. Nauk., 28(5 (173)):243-244, 1973. 